\title{
Discrimination of isotrigon textures using the Rényi entropy of Allan variances
}

\author{
Salvador Gabarda* and Gabriel Cristóbal \\ Instituto de Óptica "Daza de Valdés” (CSIC), Serrano 121, Madrid-28006, Spain \\ *Corresponding author: gabriel@optica.csic.es
}

Received January 22, 2008; revised April 25, 2008; accepted May 27, 2008; posted July 28, 2008 (Doc. ID 91961); published August 20, 2008

\begin{abstract}
We present a computational algorithm for isotrigon texture discrimination. The aim of this method consists in discriminating isotrigon textures against a binary random background. The extension of the method to the problem of multitexture discrimination is considered as well. The method relies on the fact that the information content of time or space-frequency representations of signals, including images, can be readily analyzed by means of generalized entropy measures. In such a scenario, the Rényi entropy appears as an effective tool, given that Rényi measures can be used to provide information about a local neighborhood within an image. Localization is essential for comparing images on a pixel-by-pixel basis. Discrimination is performed through a local Rényi entropy measurement applied on a spatially oriented 1-D pseudo-Wigner distribution (PWD) of the test image. The PWD is normalized so that it may be interpreted as a probability distribution. Prior to the calculation of the texture's PWD, a preprocessing filtering step replaces the original texture with its localized spatially oriented Allan variances. The anisotropic structure of the textures, as revealed by the Allan variances, turns out to be crucial later to attain a high discrimination by the extraction of Rényi entropy measures. The method has been empirically evaluated with a family of isotrigon textures embedded in a binary random background. The extension to the case of multiple isotrigon mosaics has also been considered. Discrimination results are compared with other existing methods. (c) 2008 Optical Society of America

OCIS codes: $150.1135,100.2000,100.4992$.
\end{abstract}

\section{INTRODUCTION}

Textures whose third-order correlation functions are equal to that of uniformly distributed noise patterns are denoted as isotrigon. Isotrigon patterns are a wide variety of binary (black-and-white) and ternary (black, white, and mean luminance gray) texture patterns with spatial correlation properties and reproducible characteristics [1]. In this paper, we focus on the case of binary textures only. Specifically, isotrigon textures are created by a recursion procedure in which the product of three pixels determines the value of a fourth. Textures with correlation functions based on triplets of pixels (hence trigons) have been described by Julesz et al. [1]. Systematic methods for producing isotrigon textures have been developed by Maddess et al. [2]. Purpura et al. [3] have suggested that encoding features such as edges or contours require correlations among three or more points. Isotrigon textures are useful for studying human sensitivity to these correlations. Maddess and Nagai [4] determined human discrimination performance for several classes of isotrigon textures and compared these experimental results with the outputs of two statistical discrimination models. These models employed versions of the Allan variance [5] in order to incorporate early stages of orientation processing into a scheme that examines fourth- or higher-order correlations in order to discriminate isotrigon textures. One of the models was based upon a global variance measure, and the other was based upon a localized variance with an orientation bias. To do this, they employed linear discriminant analysis (LDA) and quadratic discriminant analysis (QDA) [6]. LDA and QDA are used in statistics for determining the combination of features that better separate two or more classes of objects or events. For a detailed description of LDA and QDA, see [6]. They hypothesized that an obvious way to improve texture detection performance by these statistical methods is to increase the number of the orientations used to analyze texture images, but this would greatly increase the computational cost. However, directionality is a fundamental feature in the case of textures, and it will play a key role in our analysis. In recent years, various methods have been proposed to tackle the problem of multiorientation analysis of images [7-10].

This paper presents a new method for discriminating isotrigon textures from a binary background. The extension to the case of multitexture discrimination has also been considered. Here the discrimination is based not on the statistics of the standard autocorrelation function but on directional measurements of the entropy shown by the Allan variances of the textures. Entropy is measured through the pseudo-Wigner distribution (PWD) of local spatially oriented variances of the textures. Allan variances have shown to be an efficient preprocessing step to capture directional anisotropy when dealing with isotrigon textures [4] and have been effectively used in the experimental examples described in this paper. The PWD includes important features, being especially suitable for signal analysis, providing a straightforward pixelwise, joint space-frequency representation of a given texture, adding the possibility of being both spatially and directionally localized when used in a 1-D version. Moreover, generalized Rényi entropy measurements can provide 
quantitative criteria to evaluate the importance of the joint space-frequency information around a given pixel location.

To summarize, the aim of our method is to achieve a computational algorithm for isotrigon texture discrimination, sharing some special characteristics with the visual system, such as spatial localization, restricted spatial frequency, and orientation tuning. We do not intend for our method to reveal the way the visual system behaves; we simply take recognized mathematical tools such as the Rényi generalized entropy and the PWD to implement a workable algorithm with the already mentioned visual characteristics.

This paper is structured as follows: The basic mathematical description and theoretical background of the method are described in Section 2. Experimental results are given in Section 3. Discussions of several related aspects are presented in Section 4, and, finally, conclusions are drawn in Section 5.

\section{DESCRIPTION OF THE METHOD}

The isotrigon texture analysis method is based on three consecutive stages. The first is a processing stage that provides the Allan variance. Second, the PWD of the previous Allan variance is computed. Third, the Rényi entropy is extracted from the PWD.

\section{A. Allan Variance}

The simplest isotrigon textures have third-order statistics identical to those of coin-flip textures, i.e., textures whose pixel values are jointly independent random variables with equal probability of taking the value 0 or 1 . Globally considered (the meaning of global refers to the association of a single value of entropy for the whole image), there is no difference between the entropy of a binary noise texture and the entropy of an isotrigon texture. The classic definition of Shannon entropy, $H(S)=-\sum_{i=1}^{q} P\left(s_{i}\right) \log _{2}\left(P\left(s_{i}\right)\right)$, depends only on the probability of the different symbols and not in the way they are arranged. However, considering 2-D image data sets instead of single pixels and equating the domain types with Shannon's symbols, it can be shown [4] that the entropy is much less for isotrigon patterns. Many image transforms such as curvelets [7], countourlets [8], wedgelets [9], or directionlets [10] have been proposed to better represent the directionality that is inherent to images. By measuring the entropy in different directions, that is, by measuring the anisotropy, significant image differences can be determined. Hence, a directional entropy measure turns out to be an effective tool for identifying such differences. Isotrigon textures usually show some spatial anisotropic activity that can be revealed by extracting local and oriented variances. The Allan variance has gained some interest as a measure of characterizing time series and $1 / f^{\alpha}$ noise [5] and becomes a special case of local oriented variance when applied over 2 -D data sets. The conventional Allan variance is just the half-sum of the squared differences between adjacent points of a 1-D series of samples $x[k]$ :

$$
A V[n]=\frac{1}{2(N-1)} \sum_{k=-N / 2}^{N / 2-1}(x[n+k+1]-x[n+k])^{2}
$$

The factor $1 / 2$ ensures that if the random variables $x[k]$, $k=n-N / 2, \ldots, n+N / 2-1$, are pairwise uncorrelated, each with variance $\sigma^{2}$, then $E[A V[n]]=\sigma^{2}$. The estimator for the Allan variance [Eq. (1)] is unbiased for division by $(N-1)$ instead of $N$.

Introducing $(p, q)$ as a new notation for pixel $n$, the Allan variance can be extended to 2 -D matrices as follows:

$$
\begin{aligned}
A V_{\gamma, \lambda}[p, q]= & \frac{1}{2(N-1)^{2}} \sum_{k=-N / 2}^{N / 2-1} \sum_{l=-N / 2}^{N / 2-1}(x[p+k+\gamma, q+l+\lambda] \\
& -x[p+k, q+l])^{2} .
\end{aligned}
$$

The interpretation of Eq. (2) is actually the difference between the square contrast and the correlation [11].

Here a particular pixel $(p, q)$ represents the central one of a set of pixels $\{x[k, l]\}$ in the neighborhood. The values of $\gamma, \lambda$ are limited to those indicated in Table 1 for the only four orientations that result from considering adjacent pixels exclusively when applied to 2-D discrete images.

The nature of isotrigon textures, whose pixel values are calculated following a law that utilizes only adjacent pixels, justifies the use of a short length sequence in the calculation of the Allan variance. Figure 1 compares the Allan variance of a particular example of an isotrigon texture calculated using a horizontally oriented sample with that of a binary noise texture. Note that the Allan variance estimate (Fig. 1(d)) of the binary noise looks like a random product as well, whereas the Allan variance estimate of the isotrigon texture (Fig. 1(b)) seems to capture the anisotropy of the original (Fig. 1(a)).

\section{B. One-Dimensional Pseudo-Wigner Distribution}

Space-frequency information of a given image can be obtained by associating the gray-level spatial data to one of the well-known spatial/spatial-frequency distributions [12]. For this application, the Wigner distribution [13] has been selected. The spatial/spatial-frequency representations configure a family of functions introduced by Cohen in the 1-D signal analysis [14]. Any member of the family can be regarded as a linearly filtered version of the Wigner distribution [12]. We have based our preferences in considering the Wigner distribution as the paramount distribution among the Cohen class. Some of the properties of the Wigner distribution-such as its real character, which implicitly encodes the phase information-make it easier to handle for practical applications than other

Table 1. Possible Parameter Values in Eq. (2)

\begin{tabular}{ccc}
\hline Angle & $\gamma$ & $\lambda$ \\
\hline $0^{\circ}$ & 0 & 1 \\
$45^{\circ}$ & 1 & -1 \\
$90^{\circ}$ & 1 & 0 \\
$135^{\circ}$ & 1 & 1 \\
\hline
\end{tabular}





Fig. 1. A. Particular sample of an isotrigon texture (even zigzag) of $512 \times 512$ pixels. B. Allan variance of the given texture, using a horizontal window analysis (note that the result reveals an anisotropic nature with a privileged direction tilted $45^{\circ}$ ). C. Binary noise. D. Allan variance of the binary noise calculated in the same horizontal scheme (anisotropy is not detectable, as expected, indicating that binary noise is isotropic and all directionalities are equivalent). Variance calculations have been obtained by applying Eq. (2) for $N$ $=8$ pixels.

spatial/spatial-frequency representations that are complex.

Once the PWD has been selected apparently as the most suitable for this application, any specific pixel $n$ of the image can be associated with a vector containing its 1-D PWD, calculated in a window of length $N$. The use of a windowed 1-D transform for a 2-D signal can be justified by considering the three main aspects of the problem. First, by using a 1-D PWD, data can be arranged in any desired direction over a 2-D image; second, calculation time is greatly decreased compared to a 2 -D version of the PWD; and third, the 1-D PWD is an invertible function, and thus the information is preserved.

A discrete approximation of the Wigner distribution proposed by Claasen and Mecklembräuker [15-17], similar to Brenner's expression [18], has been chosen for this problem:

$$
W[n, k]=2 \sum_{m=-N / 2}^{N / 2-1} z[n+m] z^{*}[n-m] e^{-2 i(2 \pi m / N) k} .
$$

In Eq. (3), $n$ and $k$ represent the time- and frequencydiscrete variables, respectively, and $m$ is a shifting parameter, which is also discrete. Here $\{z[n]\}$ is a $1-\mathrm{D}$ sequence of data from the image, containing the gray values of $N$ pixels, aligned in the desired direction. Equation (3) can be interpreted as the discrete Fourier transform (DFT) of the product $z[n+m] z^{*}[n-m]$. Here $z^{*}$ indicates the complex conjugate of $z$. This equation is limited to a spatial interval $[-N / 2, N / 2-1]$ (the PWD's window), giving a local character to the information. By scanning the image with a 1-D window of $N$ pixels, i.e., by shifting the window to all possible positions over the image, the full 
pixelwise PWD of the image is produced. The window can be tilted in any direction to obtain a directional distribution.

\section{Rényi Entropy Measures}

Entropy is a measure of information in a given set of data. Since textures can be considered as representations of the surfaces of objects and it is well established that surfaces are often composed of features with multiple orientations, the question arises as to whether image-data-gatheringoriented channels transmit different amounts of information about textures and real-world images. Entropy can be applied as a global measure or as a local one, suggesting that examining the entropy at different orientations (or directions) in an image may be a useful approach to the problem of texture discrimination. The direct application of directional entropy measures can reveal orientation biases in a given texture class, but in some cases, a preprocessing step that involves the calculation of the Allan variances of the textures is required. Whether a preprocessing step is necessary or not, directional entropy measurements may be useful for detecting the anisotropy of 2-D distributions. For this reason, the Rényi entropy stands out as the most relevant entropic measure in this context.

A thorough review of the existing Rényi measures, which we summarize here, can be found in [19]. The entropy measure was initially proposed independently by Shannon [20] as a measure of the information content per symbol, coming from a stochastic information source, and by Wiener [21] as a measure of randomness. Later, Rényi [22] extended this notion to yield the generalized entropy. The use of the Rényi entropy measures in the context of time-frequency distributions was introduced by Williams et al. [23,24], with a significant contribution by Flandrin et al. [25] in establishing the properties of these measures. The Rényi entropy measure applied to a discrete space-frequency distribution $P[n, k]$ has the form

$$
R_{\alpha}=\frac{1}{1-\alpha} \log _{2}\left(\sum_{n} \sum_{k} P^{\alpha}[n, k]\right)
$$

where $n$ and $k$ represent the spatial and frequency variables, respectively; $\alpha \geqslant 2$ are values recommended for space-frequency distribution measures. Although Rényi measures of joint space-frequency distributions formally resemble the original entropies, they do not have the same properties, conclusions, and results as the classical Shannon entropy. For instance, the positivity condition $P[n, k] \geqslant 0$ will not be always preserved, along with the unity energy condition, $\Sigma_{n} \Sigma_{k} P[n, k]=1$, which are classical requirements for considering $P$ a regular probability distribution. In order to reduce a distribution to the unity signal energy case, some kind of normalization must be done [24]. The normalization can be done in various ways, leading to a variety of possible measure definitions $[19,25]$.

Quantum mechanics [26] inspires a normalizing step by associating the space-frequency distribution $P$ of a given position $n$ with a wave function. We have selected the already mentioned quantum normalization for this particular problem of isotrigon texture discrimination, due to its excellent response, as will be illustrated later in Section 3.

A wave function is a mathematical tool used in quantum mechanics to describe any physical system. The values of the wave function are complex numbers that, when multiplied by their complex conjugates, give the probability distribution of the possible states of the system. In a similar way, we may derive a probability distribution by interpreting $W[n, k]$ in Eq. (3) as the wave function of the pixel states.

In such a case, $\breve{W}[n, k]=W[n, k] W *[n, k]$ is the probability distribution of $W$. Here $W^{*}$ represents the complex conjugate of $W$. A normalizing step must follow to satisfy the normalizing condition $\Sigma_{n} \Sigma_{k} \breve{W}[n, k]=1$.

The general case in Eq. (4) with $\alpha=3$, identifying $P$ with the normalized PWD [ $W$ in Eq. (3)], and interpreting the measure on a pixelwise basis, leads us to

$$
R_{3}[n]=-\frac{1}{2} \log _{2}\left(\sum_{k} \breve{W}^{3}[n, k]\right) .
$$

Here $W$ has been normalized by

$$
\breve{W}[n, k]=\frac{W[n, k] W^{*}[n, k]}{\sum_{k}\left(W[n, k] W^{*}[n, k]\right)} .
$$

An accurate uncertainty measure, especially in situations when position and momentum are highly correlated, has been proposed by Süßmann [27], known as the RényiSüßmann entropy. Süßmann proposed an entropy measure that employs the Wigner distribution after squaring the distribution values [28]. Dealing with images, the typical variables are position and frequency. Images, considered as random processes, show a high correlation between position and frequency in neighborhood pixels, the exception being made at the borders of the objects in the image. These properties have to be considered when an appropriate model has to be built. The Wigner distribution was proposed by Wigner as a phase-space representation in quantum mechanics [13]. Subsequently, Ville derived in the area of signal processing the same distribution that Wigner derived some years before [29]. After that, phase-space methods became widespread in an increasing number of applications due mainly to their intuitive character and to their being comprehensively broad [30].

\section{EXPERIMENTAL RESULTS}

The tests that are going to be described below have been performed by generating texture samples at random each time with a MATLAB function due to Maddess [4]. We assume that there is enough image diversity, based in the number of trials and in the random differences among the samples used in every particular experiment. Also, the method previously described involves several parameters that must be set up to have accurate results. Anisotropy may present many different schemes. In particular, isotrigon textures involve a strong correlation among neighboring pixels. Presumably, the window size of the PWD can be small and has been fixed after some experimental 
trade-off with the results. Orientations are restrained to the directionalities available with the Allan variance.

The discriminating process requires a test image and two reference images. The test image contains the target isotrigon texture embedded in a binary random background. One of the reference images is an arbitrary sample of the isotrigon texture under test; the second reference image is an arbitrary sample of the binary random noise in the background of the test image. In the examples that follow, several digital images of 512 $\times 512$ pixels with 8 bits/pixel have been considered. The three previously mentioned images are subjected to a preprocessing step consisting of substituting their original pixel values for their local Allan variances calculated according to Eq. (2) for $N=8$ and using the right orientation, which is determined empirically (as described below). The four possible orientations, $0^{\circ}, 45^{\circ}, 90^{\circ}$, and $135^{\circ}$, will be referred as $\mathrm{AV}_{0}, \mathrm{AV}_{45}, \mathrm{AV}_{90}$, and $\mathrm{AV}_{135}$, respectively. Also, combinations of the variances are examined by squaring sums and differences. The results are biased to have a minimum value over zero $(+1$ was determined to be a suitable bias by trial and error). Biasing the values is not a trivial operation, because the PWD and the Rényi entropy are nonlinear operations and the result depends upon the range in which the values are set. Hence, biasing the values influences the sensitivity of the process. It is not critical, but operations with all values close to zero are not recommendable.

Allan variances are then submitted to a PWD calculation process to obtain their joint spatial-frequency pixelwise distributions. The PWDs have been taken using a 1-D scheme and calculated by a sliding window of $N$ $=8$ pixels, as indicated by Eq. (3), and oriented according to the spatial anisotropy shown by the Allan variances of the textures. The PWD is oriented to have a minimum of the Rényi entropy. Sometimes the orientation is visible (e.g., $45^{\circ}$ for even zigzag texture in Fig. 1); in other cases, measures must be compared among the possible orienta-
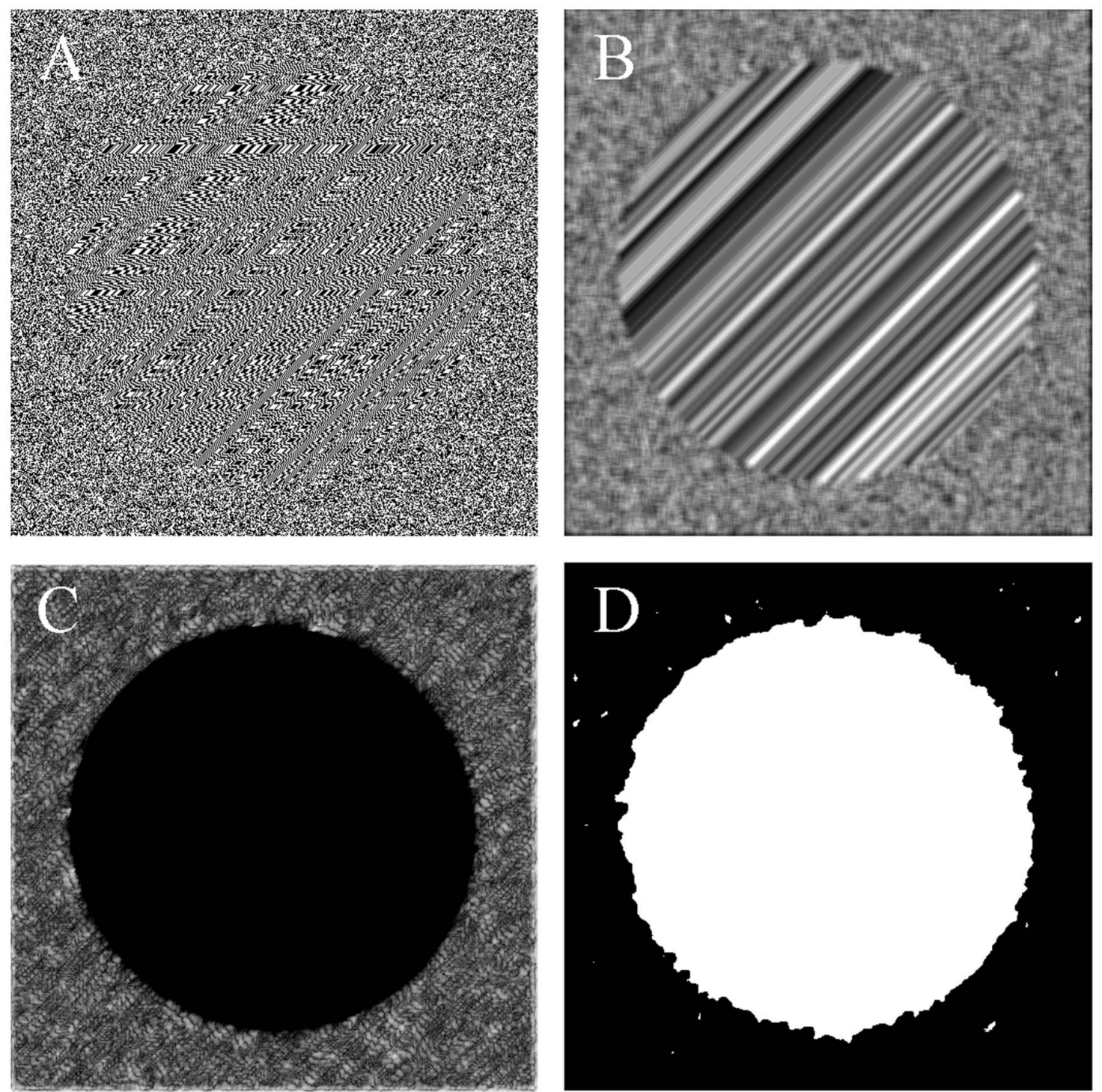

Fig. 2. Example of detection of a test texture (even zigzag) of $512 \times 512$ pixels. A. Texture embedded in a binary random noise background. B. Result of the application of the Allan variance to A with an horizontal orientation ( $N=8$ pixels). C. Entropy map. D. DM after applying the Rényi entropy over a PWD of B tilted $45^{\circ}$ and a window of $N=8$ pixels. 
Table 2. Discrimination Performance ${ }^{a}$

\begin{tabular}{|c|c|c|c|c|c|c|c|c|c|c|}
\hline \multirow[b]{2}{*}{ Texture } & \multicolumn{5}{|c|}{ Even } & \multicolumn{5}{|c|}{ Odd } \\
\hline & $\mathrm{AV}$ & PWD & $\mathrm{S}$ & $\mathrm{Sp}$ & S\&Sp & $\mathrm{AV}$ & PWD & $\mathrm{S}$ & $\mathrm{Sp}$ & S\&Sp \\
\hline $1 \mathrm{Box}$ & $\mathrm{AV}_{0}$ & $90^{\circ}$ & 0.9548 & 0.9986 & 0.9767 & $\mathrm{AV}_{0}$ & $0^{\circ}$ & 0.9747 & 0.9960 & 0.9853 \\
\hline 2 Triangle & $\left(\mathrm{AV}_{45}-\mathrm{AV}_{90}\right)^{2}$ & $90^{\circ}$ & 0.9490 & 0.9967 & 0.9729 & $\left(\mathrm{AV}_{45}-\mathrm{AV}_{90}\right)^{2}$ & $90^{\circ}$ & 0.9637 & 0.9893 & 0.9765 \\
\hline 3 Cross & $\mathrm{AV}_{45}$ & $135^{\circ}$ & 0.9755 & 0.9894 & 0.9825 & $\left(\mathrm{AV}_{45}-\mathrm{AV}_{135}\right)^{2}$ & $45^{\circ}$ & 0.9602 & 0.9840 & 0.9723 \\
\hline 4 Zigzag & $\mathrm{AV}_{0}$ & $45^{\circ}$ & 0.9698 & 0.9955 & 0.9827 & $\mathrm{AV}_{45}$ & $0^{\circ}$ & 0.9663 & 0.9950 & 0.9807 \\
\hline 5 Oblong & $\mathrm{AV}_{90}$ & $0^{\circ}$ & 0.9657 & 0.9931 & 0.9794 & $\mathrm{AV}_{90}$ & $90^{\circ}$ & 0.9742 & 0.9964 & 0.9853 \\
\hline 6 Tee & $\left(\mathrm{AV}_{0}-\mathrm{AV}_{45}\right)^{2}$ & $0^{\circ}$ & 0.9654 & 0.9823 & 0.9738 & $\left(\mathrm{AV}_{45}-\mathrm{AV}_{135}\right)^{2}$ & $90^{\circ}$ & 0.9565 & 0.9943 & 0.9754 \\
\hline 7 Wye & $\left(\mathrm{AV}_{45}-\mathrm{V}_{135}\right)^{2}$ & $0^{\circ}$ & 0.9451 & 0.9966 & 0.9709 & $\left(\mathrm{AV}_{45}+\mathrm{AV}_{135}\right)^{2}$ & $0^{\circ}$ & 0.9700 & 0.9874 & 0.9787 \\
\hline 8 Foot & $\left(\mathrm{AV}_{45}-\mathrm{AV}_{90}\right)^{2}$ & $90^{\circ}$ & 0.9561 & 0.9959 & 0.9760 & $\left(\mathrm{AV}_{45}+\mathrm{AV}_{90}\right)^{2}$ & $90^{\circ}$ & 0.9684 & 0.9917 & 0.9801 \\
\hline $9 \mathrm{El}$ & $\left(\mathrm{EV}_{0}-\mathrm{AV}_{90}\right)^{2}$ & $90^{\circ}$ & 0.9433 & 0.9624 & 0.9529 & $\left(\mathrm{AV}_{0}+\mathrm{AV}_{90}\right)^{2}$ & $90^{\circ}$ & 0.9624 & 0.9742 & 0.9683 \\
\hline
\end{tabular}

${ }^{a}$ Textures used to determine the quality of the method, indicating the best directionalities observed and the maximum accuracy obtained for S\&Sp, according to the threshold values that are determined after the ROC process. S, sensitivity; Sp, specificity.

tions. Once the Rényi entropies of the pixelwise distributions have been measured, a decision map (DM) that determines the texture discrimination is derived.

The DM is derived as follows. Every pixel in the image has a different $N$-component PWD vector associated with it; the amount of information related to a given pixel of the image is measured by the Rényi entropy of this PWD, according to Eq. (5). Distances can be measured pixel to pixel by using the Rényi entropies associated with the test and the reference images, i.e.,

$$
D_{i}[n]=\left\|\left\langle R_{i}\right\rangle-\left\langle R_{T}[n]\right\rangle_{N}\right\|, \quad i \in\{1,2\} .
$$

Here $R_{i}$ is a matrix indicating the Rényi entropy of a given reference texture $i$ ( $i=1$ noise sample, $i=2$ texture sample) in a pixelwise scheme. Brackets indicate mean values corresponding to the samples of the textures. Finally, $\left\langle R_{T}[n]\right\rangle_{N}$ indicates the mean value of the Rényi entropy measured in the test image, calculated in the neighborhood of $N \times N$ pixels around the position $n$. The maximum of $\left\{D_{1}[n], D_{2}[n]\right\}$ is used to determine the DM:

$$
D M[n]=\underset{i}{\arg \max }\left(D_{i}[n]\right), \quad i \in\{1,2\} .
$$

As only two results are possible, a binary DM is derived. The white pixels can be set to indicate the location of the isotrigon texture and the black ones to indicate the binary noise. Figure 2 shows an example of a particular texture. Here a $45^{\circ}$ tilted PWD of $\mathrm{AV}_{0}$ has been used to measure the entropy.

The algorithm can be summarized as indicated below:

Three images are involved: test image (TI), texture reference (TR), and noise reference (NR). For each image, perform the following steps:

(1) Take a pixel $n$.

(2) Consider the neighborhood of pixel $n$, consisting in $N$ pixels centered in $n$ and aligned in any of the four possible directions, $0^{\circ}, 45^{\circ}, 90^{\circ}$, and $135^{\circ}$.

(3) Use Eq. (2) to calculate the four Allan variances and their derived squared sums and differences (see Table 2), associated with pixel $n$.

(4) Repeat the calculations with all the pixels in the three input images.

(5) Use Eq. (3) to calculate the PWDs of all the AVs obtained above, using the same set of orientations.
(6) Draw a set of DMs by considering all the related products (TI, TR, and NR after the same combination of AVs and PWDs) by means of Eqs. (5)-(7).

(7) Select the most discriminating DM to perform the segmentation.

The method has been applied to a set of isotrigon textures, of $512 \times 512$ pixels in size, to determine its discrimination capabilities. The textures used in this experimental section belong to the nine families of isotrigon textures tested by Maddess and Nagai [4]. Each member of the nine families contains two version's termed even and odd. Therefore, the total number of cases considered is 18. The directionality of the different textures was discovered after applying systematically all the possible Allan variances (or combinations) of them. Squaring of the sums and differences of the $\mathrm{AVs}$ has been used when simple Allan variances did not show a directional preference. An example of this situation is illustrated in Fig. 3.

This experiment has been statistically evaluated by considering 10 different samples of each of the 18 different isotrigon textures selected. The local analysis has been performed using a window of $8 \times 8$ pixels in the neighborhood of the target pixel to evaluate the Allan variance $(N=8)$. Also a 1 -D windowing scheme of $N$ $=8$ pixels has been used to evaluate the Rényi entropy through the oriented 1-D PWDs. The segmentation performance has been determined by comparing the DM with the mask used to build the test samples. The quality parameter used has been the percentage of correct decision (PCD), defined as

$$
P C D=100 \times \frac{\text { number of accurate pixels }}{\text { total number of pixels }} .
$$

Texture and noise areas have been computed separately. Two indices have been derived from the PCD measured in the DM, considering the two different areas (texture and noise) independently. The first one is called sensitivity (S) and is defined as the probability of correctly recognizing an isotrigon field as a texture. The other is called specificity ( $\mathrm{Sp}$ ) and is defined as the probability of reporting random when the texture was random. The results have been interpreted through a receiver operating characteristic (ROC) by plotting the sensitivity versus (1-specificity). The last one is usually 

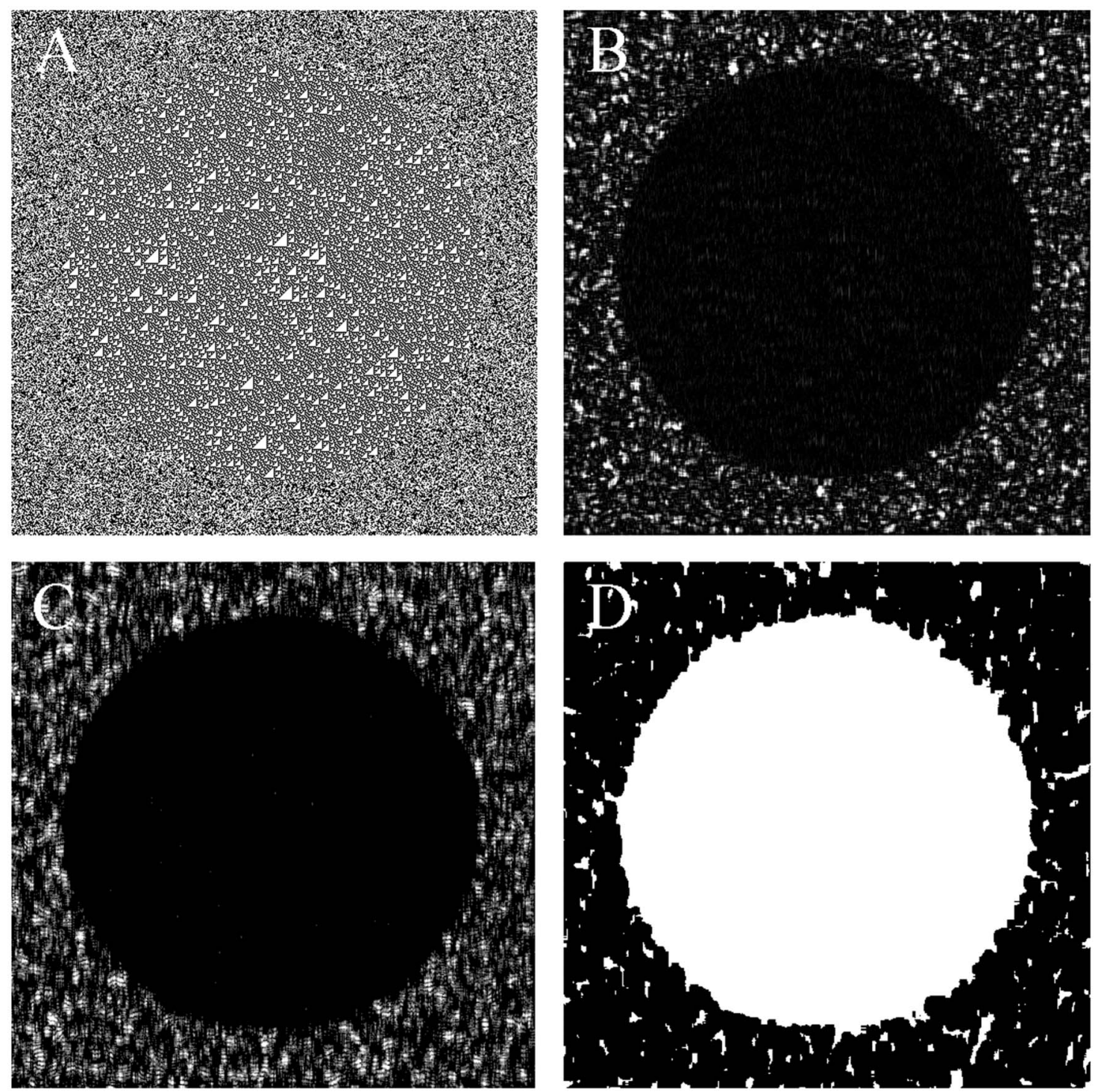

Fig. 3. Example of detection of a isotrigon texture (even triangle) by squaring of the differences of Allan variances. A. Target isotrigon texture embedded in a binary random noise background. B. Squaring of Allan variances $\left(\mathrm{AV}_{45}-\mathrm{AV}_{0}\right)^{2}$ obtained from $\mathrm{A}(N=8$ pixels $)$. C. Entropy map. D. DM after applying the Rényi entropy, using a PWD tilted $90^{\circ}$ and a window of $N=8$ pixels.

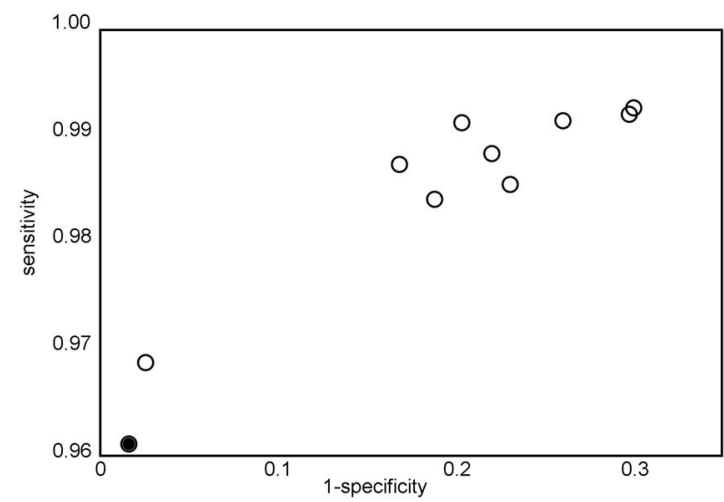

Fig. 4. Example of ROC for the odd cross texture versus random noise. The highest simultaneous quality is selected to determine the threshold values, i.e., $\left\langle R_{i}\right\rangle$ values in Eq. (9). The experiment indicated by a black circle provides the best threshold values to discriminate this texture from binary noise, using the directionalities indicated in Table 2 for this texture.

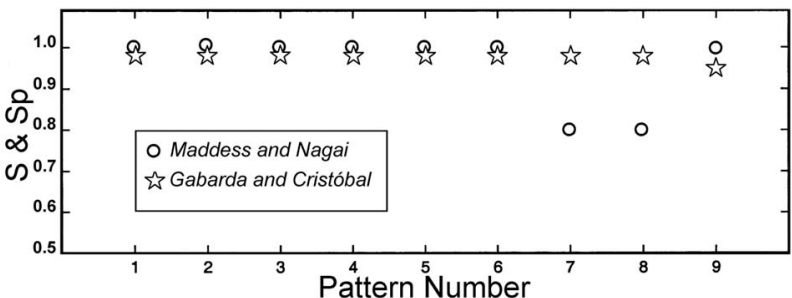

Fig. 5. Comparison of the results of this method for nine families of even isotrigon textures versus the most relevant QDA results given by Maddess and Nagai [4].

referred to as the false positive rate. This plotting is normally used for binary classifier systems when the discrimination threshold is varied. Figure 4 shows the ROC for the odd cross texture versus random noise. The highest simultaneous quality performance is selected (S\&Sp) to determine the threshold values [i.e., $\left\langle R_{i}\right\rangle$ values in Eq. 



Fig. 6. A. Image consisting of five isotrigon textures. B. Segmentation of region "0" (even oblong). C. Segmentation of region "1" (even triangle). D. Segmentation of region "2" (even zigzag). E. Segmentation of region "3" (even cross). F. Segmentation of region "4" (even box). Regions " 2 , 3, and 4" can be segmented straightforwardly by using the directionalities indicated in Table 2 . Region " 0 " has the same entropy for the parameters given in Table 2 as region "4" but has been isolated by difference with this one. Region " 1 " has been obtained by eliminating the other four, as no suitable directionality was observed. G. DM after previous single results. H. DM by a complex wavelets method [33,34]. Figure 6(h) courtesy of M. G. Forero.

(6)] as, for example, the experimental result indicated with a black circle in Fig. 4 . Note that to discriminate a texture under test, we can choose among an infinitely diverse number of texture and noise representatives to be compared with the test texture. Which texture and noise samples must be used is decided by the ROC process, selecting among all the experiments the one with the maximum S\&Sp value. Table 2 shows the expected quality per- formances when using the optimal threshold values previously determined, after applying the method described here to the same set of test textures used by Maddess and Nagai [4]. Results for even isotrigon textures versus binary random noise are compared in Fig. 5 with the most relevant QDA referenced by [4].

The method can be extended to the case of discriminating multiple isotrigon textures. For example, Fig. 6 pre-
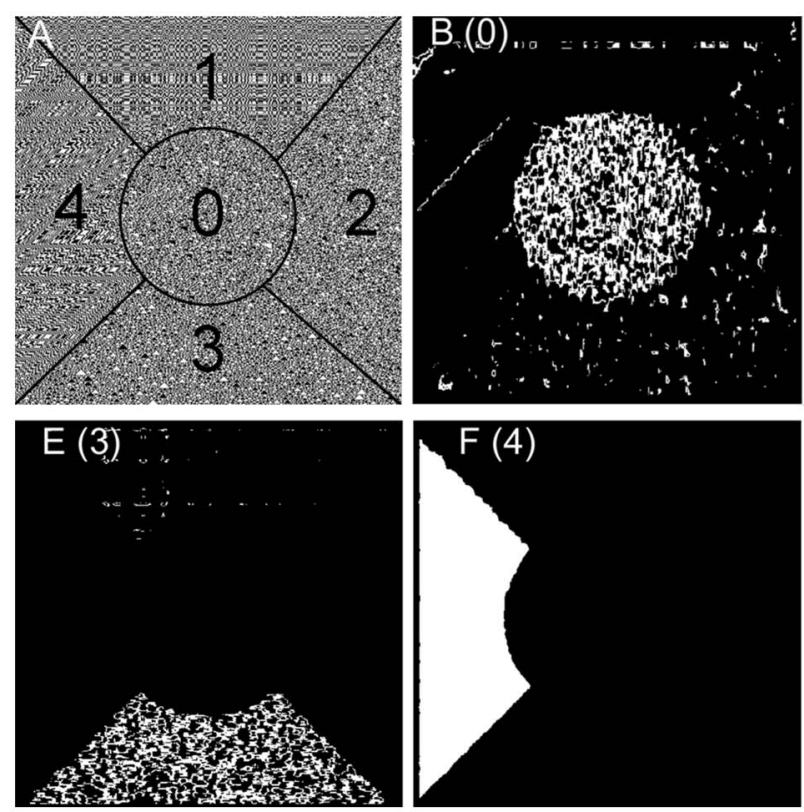
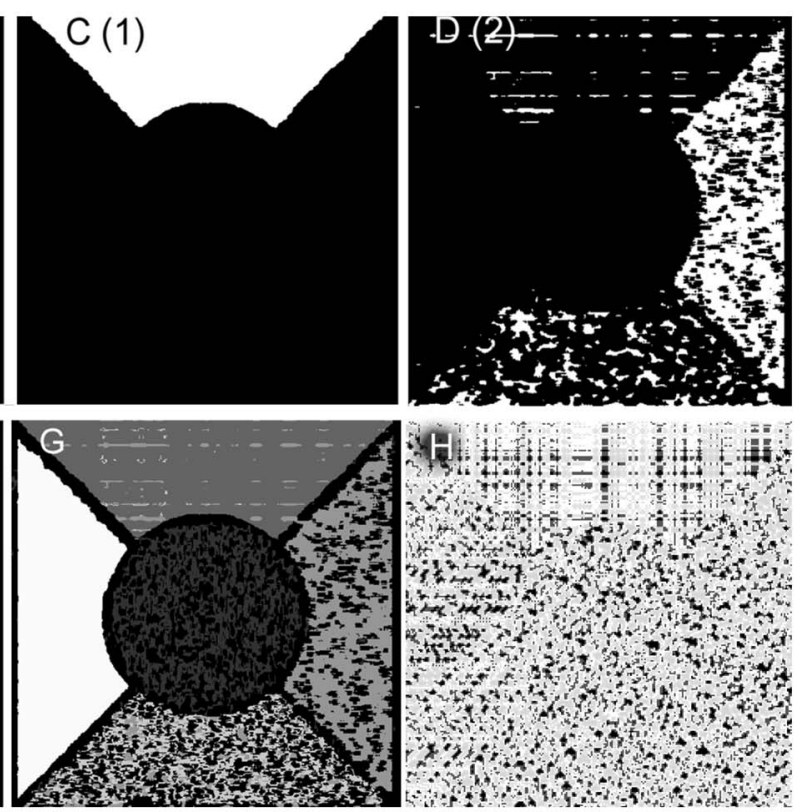

Fig. 7. A. Image consisting of five isotrigon textures. B. Segmentation of region "0" (even El). C. Segmentation of region "1" (even oblong). D. Segmentation of region "2" (even wye). E. Segmentation of region "3" (even tee). F. Segmentation of region "4" (even zigzag). All regions can be segmented straightforwardly by using the directionalities indicated in Table 2 . Only regions " 1 " and "4" have been perfectly identified. Regions “ $0,2,3$, and 4" have been recognized with some degree of uncertainty. G. DM after previous single results. H. DM by a complex wavelets method [33,34]. Figure 7(h) courtesy of M. G. Forero. 
Table 3. Percentage of Correct Decisions [PCD, Eq. (8)] of Isotrigon Even Texture Pairs Corresponding to Three Different Segmentation Methods

\begin{tabular}{|c|c|c|c|c|}
\hline Texture Pair & Markov Method [35] & Gabor Filtering Method [35] & $\begin{array}{c}\text { Fractal } \\
\text { Dimension } \\
{[35]}\end{array}$ & $\begin{array}{c}\text { Allan/Rényi } \\
\text { Method } \\
\text { (This Paper) }\end{array}$ \\
\hline Cross-Zigzag & 100 & 87 & 68 & 92 \\
\hline Zigzag-Oblong & 88 & 81 & 58 & 91 \\
\hline Zigzag-El & 87 & 81 & 65 & 88 \\
\hline Cross-El & 71 & 81 & 59 & 88 \\
\hline Mean & 86 & 82 & 62 & 89 \\
\hline
\end{tabular}

sents a mosaic of five different textures. Using the discrimination directions indicated in Table 2 for these textures and the algorithm previously described, textures have been segmented. Figures 6(b)-6(f) present the segmentation results for such isotrigon mosaics. The input to the algorithm consists of a set of the 18 isotrigon textures considered (9 even and 9 odd) and the test image " $\mathrm{A}$ " that appears at the top left corner of Fig. 6 . The result is a labeled DM indicating the position where every texture matches the entropy of the test image. Differences in entropy between textures are not always sufficiently discriminative, hence textures as, for example, even triangle (Fig. 6(a), region 1) cannot be segmented, because the entropy measured is similar to the entropy measured in other regions. Hence, it requires segmentation by elimination when the other regions have been already recognized. Also, coincidence of entropy in a given Allan combination is possible, as, for example, even oblong (region 0 ) and even box (region 4). In this case, region " 0 " has been isolated by subtracting region 4 . Figure 7 presents another example of multitexture isotrigon discrimination. Here regions 1 and 4 are clearly defined, but the remaining regions present a certain amount of uncertainty. Single discrimination results have been combined to draw the complete DMs for these examples (i.e., Figs. 6(g) and $7(\mathrm{~g})$ ). A quantitative comparative study of the classification accuracy has been performed by considering texture pairs as shown in Table 3. One alternative method chosen was a Gabor filtering technique optimally designed through a genetic algorithm for best performance in texture segmentation [31,32]. Each Gabor filter has been tuned to each texture to satisfy maximum discrimination, where a preprocessing stage based on a second-order Shannon entropy has been considered for better performance. This study does not pretend to be exhaustive and only illustrates the limitations of other traditional methods in the discrimination of those high-order textures. Table 3 shows that the above-mentioned Gabor-based filtering method has been outperformed by the method introduced in this paper for the textures indicated in the table. A similar performance can be expected for other texture pairs, including even/odd combinations. On the other hand, it is worth mentioning that the current method can produce a few false positives located in the background (see, e.g., Fig. 3(c)), which presumably could be avoided by means of some modification of the method that has not been investigated yet. For example, one could use a measure other than $\max \left\{D_{1}[n], D_{2}[n]\right\}$, such as a threshold difference, and then derive the ROCs by varying the threshold. However, the max criterion combines the advantages of its simplicity and accuracy. In addition to that, some postprocessing could be applied for eliminating isolated points, such as through median filtering. On the other hand, methods based on multiscale decompositions such as the complex wavelets failed to give an acceptable DM, as revealed by Figs. 6(h) and 7(h), which show the results obtained with such a technique [33,34].

The current method has been compared with the MeasTex test suite, which is a framework for quantitative comparisons of texture classification algorithms [35]. Three different texture classification algorithms have been considered in this study: Gauss random Markov field, Gabor energies, and fractal dimension (see Table 3). Gray-level co-occurrence matrices were not included in this study due to the bilevel characteristics of the isotrigon textures. Of all competing approaches, the Markov-based method performs the best. However, the current method outperforms other existing methods for the mean texture data set (see Table 3).

\section{DISCUSSION}

We have implemented a biological-inspired workable algorithm capable of discriminating isotrigon textures. Our method mimics some simple properties of the visual system such as spatial/spatial-frequency localization and orientation tuning, and it displays some parallelism with the nonlinearities that have been observed in the visual pathways. Tyler [36] and later Schumer and Ganz [37] corroborated that the human brain is performing spatialfrequency analysis as an initial stage before pattern recognition takes place. In this paper, discrimination of ensembles of isotrigon textures is attained using fourthorder correlation approaches as described in [3]. Experimental results due to Purpura et al. [3] show that there exist mechanisms in the monkey visual cortex that can discriminate between textures differing in certain fourthorder spatial correlations. Purpura et al. [3] concluded that the visual cortex must have mechanisms capable of detecting spatial correlations involving three or more points in the image. Linear spatiotemporal filtering cannot detect spatial correlations higher than second order. However, oriented filters, combined with a special set of nonlinearities, may be a key computational component in the cortex area V1 for detecting these higher-order correlations. Thus, the proposed method can be regarded as a 
computational model that implements some essential salient features of the visual system, such as nonlinearity, orientation selectivity, multiple spatial correlation mechanisms, and spatial/spatial-frequency localization.

We now consider the rationale behind our approach. The rationale of the method described here is to implement such fourth-order mechanisms through the combined use of the Allan variance and the PWD computation. The Allan variance constitutes a neurally plausible way to implement quadratic interactions [3]. The PWD analysis provides a quadratic nonlinearity order as well. Therefore our method provides a fourth-order spatial correlation approach, which appears to be among the earliest information extracted by our visual brain and that constitutes the basic core mechanism for isotrigon texture discrimination [38]. Some criticism can be raised about the optimal window size to be used in the experiments. The window size is a compromise among spatial-frequency contents, spatial localization, and computational complexity. The local entropy associated to each pixel is computed using a 1-D-oriented window of 8 pixels, extracted from an $8 \times 8$ neighborhood of each pixel, and following a previously selected orientation. It could be possible that the optimization of the parameters involved in the algorithm should originate an even better discrimination, but this study is beyond the scope of the present paper. Thus, the number of pixels used in our experiments has been chosen following an empirical procedure. Unless the local domain is $8 \times 8$ pixels, calculations at the pixel level are performed using a 1-D-oriented window of 8 pixels. Hence, only 8 pixels are used to determine the entropy of a given pixel and consequently to establish the discrimination measure. The number of pixels used is an even number according to the definition assumed for the PWD. The complexity of the calculations can be reduced by using smaller windows, but the accuracy of the method is not expected to be better. Herein, the pixel entropy is associated with spatial frequencies. Localized spatial frequencies are measured by considering a neighborhood of pixels. In such a sense, there is always an implicit configuration of pixels, i.e., the oriented window used to perform the calculations.

In summary, our method is a texture discrimination method tuned exclusively for isotrigon ensambles. It has been designed as a "blind method," because it ignores how the textures have been generated. Hence, the problem of identifying the textures when generating rules are known in advance is not an issue here. A comparison with other texture discrimination methods has been presented. We have selected the set of textures already studied by Maddess and Nagai [4] in order to have a qualitative and quantitative reference with our results. The examples presented indicate that sharp boundaries are obtained for noise-texture discrimination and comparable to the size of the window in the texture mosaics (see Figs. 6 and 7).

\section{CONCLUSIONS}

A new method for discriminating isotrigon textures versus binary noise and isotrigon textures of different types has been developed and evaluated. This new method is based in a particular Rényi entropy measure, through the
Allan variances of the textures. The method has been evaluated by means of a systematic study applied to 18 different textures, showing that this method makes possible a high discrimination performance similar to and in some cases better than other known methods such as QDA. The method has been extended to the multitexture discrimination task. Results have shown that the method provides a good discrimination performance even in such cases. Although the multitexture discrimination problem has not yet been completely solved by this technique, the results are significantly better than other discrimination methods, such as wavelets, indicating that further research based in this approximation may lead to a full discrimination of isotrigon multitextures.

Also, this method provides new avenues to deal with the problem of discriminating high-order textures in general and, more important, shares relevant characteristics with the visual system. Some of the most commonly recognized properties of cortical cells are spatial localization, restricted spatial frequency, and orientation tuning. Our method relies on these properties, and it provides a fourth-order correlation procedure. Although its psychophysical validation is beyond the scope of the present paper, it opens up new research directions to analyze the psychophysical response of the Rényi directional entropies for the discrimination of isotrigon stimuli. Comprehensive comparisons with other segmentation techniques are left for further work. In addition to that, further research is required to analyze the new trilevel isotrigon textures developed by Maddess et al. [2]. Also, comparative measures other than the sensitivity and the specificity that have been used here can be considered as valuable.

\section{ACKNOWLEDGMENTS}

This research has been supported by the following projects: TEC2004-00834, TEC2005-24739-E, TEC200524046-E, and 20045OE184 sponsored by the Spanish Ministry of Science; and PI040765 sponsored by the Spanish Ministry of Health. We thank Ted Maddess for fruitful discussions and for providing us with the MATLAB code to generate the isotrigon textures presented in this paper. We thank Manuel Forero and M. E. Barilla for providing us with Figs. $6 \mathrm{H}$ and $7 \mathrm{H}$.

\section{REFERENCES}

1. B. Julesz, E. N. Gilbert, and J. D. Victor, "Visual discrimination of textures with identical third-order statistics," Biol. Cybern. 31, 137-140 (1978).

2. T. Maddess, Y. Nagai, A. C. James, and A. Ankiewcz, "Binary and ternary textures containing higher-order spatial correlations," Vision Res. 44, 1093-1113 (2004).

3. K. P. Purpura, J. D. Victor, and E. Katz, "Striate cortex extracts higher-order spatial correlations from visual textures," Proc. Natl. Acad. Sci. U.S.A. 91, 8482-8486 (1994).

4. T. Maddess and Y. Nagai, "Discriminating isotrigon textures," Vision Res. 41, 3837-3860 (2001).

5. D. W. Allan, "Statistics of atomic frequency standard," Proc. IEEE 54, 223-231 (1966).

6. R. A. Johnson and D. W. Wichern, Applied Multivariate Statistical Analysis (Prentice Hall, 1992).

7. J. L. Starck, E. Candes, and D. Donoho, "The curvelet 
transform for image denoising," IEEE Trans. Image Process. 11, 670-684 (2002).

8. M. N. Do and M. Vetterli, "The contourlet transform: an efficient directional multiresolution image representation," IEEE Trans. Image Process. 14, 2091-2106 (2005).

9. D. Donoho, "Wedgelets: nearly minimax estimation of edges," Ann. Stat. 27, 859-867 (1999).

10. V. Velisavljevic, B. Beferull-Lozano, M. Vetterli, and P. L. Dragotti, "Directionlets: anisotropic multidirectional representation with separable filtering," IEEE Trans. Image Process. 15, 1916-1933 (2006).

11. A. Cumani, "Edge detection in multispectral images," Comput. Vis. Graph. Image Process. 53, 40-51 (1991).

12. L. D. Jacobson and H. Wechsler, "Joint spatial/spatialfrequency representation," Signal Process. 14, 37-68 (1988).

13. E. Wigner, "On the quantum correction for thermodynamic equilibrium," Phys. Rev. 40, 749-759 (1932).

14. L. Cohen, "Generalized phase-space distribution functions," J. Math. Phys. 7, 781-786 (1966).

15. T. A. C. M. Claasen and W. F. G. Mecklenbräuker, "The Wigner distribution-a tool for time-frequency analysis. Part I. Continuous-time signals," Philips J. Res. 35, 237-250 (1980).

16. T. A. C. M. Claasen and W. F. G. Mecklenbräuker, "The Wigner distribution-a tool for time-frequency analysis. Part II. Discrete-time signals,” Philips J. Res. 35, 276-300 (1980).

17. T. A. C. M. Claasen and W. F. G. Mecklenbräuker, "The Wigner distribution-a tool for time-frequency analysis. Part III. Relations with other time-frequency transformations," Philips J. Res. 35, 372-389 (1980).

18. K. H. Brenner, "A discrete version of the Wigner distribution function," EURASIP J. Appl. Signal Process. 2005, 307-309.

19. L. Stankovic, "A measure of some time-frequency distributions concentration," Signal Process. 81, 623-631 (2001).

20. C. E. Shannon and W. Weaver, The Mathematical Theory of Communication (University of Illinois Press, 1949).

21. N. Wiener, Cybernetics (Wiley, 1948).

22. A. Rényi, "Some fundamental questions of information theory," in Selected Papers of Alfréd Rényi, P. Turán, ed. (Akadémiai Kiadó, 1976), pp. 526-552 (1976). [Originally published in Magy. Tud. Akad. Mat. Fiz Tud. Oszt. Kozl., 10, 251-282 (1960)].

23. W. J. Williams, M. L. Brown, and A. O. Hero, "Uncertainty, information and time-frequency distributions," Proc. SPIE 1566, 144-156 (1991).

24. T. H. Sang and W. J. Williams, "Rényi information and signal dependent optimal kernel design." in Proceedings of the IEEE International Conference on Acoustics, Speech, and Signal Processing (IEEE, 1995), Vol. 2, pp. 997-1000.

25. P. Flandrin, R. G. Baraniuk, and O. Michel, "Time-frequency complexity and information," in Proceedings of the IEEE International Conference on Acoustics, Speech, and Signal Processing (IEEE, 1994), Vol. 3, pp. 329-332.

26. R. Eisberg and R. Resnick, Quantum Physics (Wiley, 1974).

27. G. Süßmann, "Uncertainty relation: from inequality to equality," Z. Naturforsch. 52, 49-52 (1997).

28. J. J. Wlodarz, "Entropy and Wigner distribution functions revisited," Int. J. Theor. Phys. 42, 1075-1084 (2003).

29. J. Ville, "Théorie and applications de la Notion de Signal Analytique," Cables Transm. 2A, 61-74 (1948).

30. D. Dragoman, "Applications of the Wigner distribution function in signal processing," EURASIP J. Appl. Signal Process. 10, 1520-1534 (2005).

31. B. Yegnanarayana, P. Pavan Kumar, and S. Das, "Onedimensional Gabor filtering for texture edge detection," Proceedings of Indian Conference on Computer Vision, Graphics and Image Processing (IEEE, 1998), pp. 231-237.

32. MATLAB code for segmentation and classification of multitexture images is available from http:// www.cse.iitk.ac.in/ amit/courses/768/00/rajrup/.

33. P. de Rivaz and N. Kingsbury, "Complex wavelet features for fast texture image retrieval," Proceedings of IEEE Conference on Image Processing (IEEE, 1999), pp. 25-28.

34. M. E. Barilla, M. G. Forero, and M. Spann, "Color-based texture segmentation," in Information Optics, 5th International Workshop, G. Cristóbal, B. Javidi, and S. Vallmitjana, eds. (AIP, 2006), pp. 401-409.

35. G. Smith and I. Burns, "MeasTex image texture database and test suite" (1997). Available online at http:// www.texturesynthesis.com/meastex/meastex.html.

36. C. W. Tyler, "Stereoscopic tilt and size effects," Perception 4, 287-192 (1975).

37. R. Schumer and L. Ganz, "Independent stereoscopic channels for different extents of spatial pooling," Vision Res. 19, 1303-1314 (1979).

38. T. Maddess and Y. Nagai, "Lessons from biological processing of image texture," in International Congress Series (Elsevier, 2004), Vol. 1269, pp. 26-29. 